\title{
Briefe des St. Galler Arztes David Christoph Schobinger an Albrecht von Haller

\author{
Von Carl Wegelin, St. Gallen
}

Im Anschluß an die Briefe des St.Galler Stadtarztes Peter Giller an HALleR, welche ich auszugsweise im 7.Jahrgang des «Gesnerus» (Heft1) veröffentlicht habe, soll hier über die Briefe berichtet werden, welche GILLers Neffe David Christoph Schobinger in den Jahren 1746-1760 an HaLler gerichtet hat (Briefsammlung der Berner Stadtbibliothek ${ }^{1}$ ).

Das Geschlecht der Schobinger stammt urkundlich aus Wil (St. Gallen). Der Zweig, welchem der Briefschreiber angehörte, wurde 1520 mit HaNS Schobinger in der Stadt St.Gallen eingebürgert. Dessen gelehrter Sohn BARTHOLOME gelangte als Eisenhändler zu großem Reichtum und wurde in die adelige Innung der Notensteiner aufgenommen. Bartholomes Sohn ToBIAS, in Mathematik und Architektur bewandert, hatte neun Söhne, die wie ihr Vater in ganz Europa herumreisten und sehr sprachenkundig waren. Sie erhielten 1623 von Kaiser Ferdinand II. einen Adelsbrief, in welchem die Sсновinger als ein «uralt adeliges Geschlecht» bezeichnet werden. Unter diesen Söhnen war Sebastian (1579-1652) der bedeutendste (siehe sein Lebensbild von P.Jung). Er war zuerst Leibarzt des späteren Kaisers Mathias, dann Stadtarzt und Arzt des Stiftes in St.Gallen, durch seine Kunst in der ganzen Ostschweiz berühmt und von 1632-1652 Bürgermeister der Stadt St. Gallen.

Im 17. und 18. Jahrhundert waren noch mehrere Sсновinger Stadtärzte in St.Gallen. So David (1594-1618), Heinrich (1602-1642), Jeremias (1625-1673), Bartholome (1610-1675) und Johann Caspar (1701-1763). Heinrich Schobinger studierte in Padua und war Famulus von Fabricius Hildanus in Bern, wurde 1633 Malzeyschauer (Malzey=Lepra) und 1635 Stadtarzt in St.Gallen. Joh. Caspar Schobinger studierte mit Haller in Paris. Von ihm ist in der Berner Stadtbibliothek ein vom 17. September 1730 datierter Brief erhalten, den er auf der Heimreise von Paris von Königsfelden aus an HaLler schrieb und in welchem er diesen um sein ferneres Wohlwollen bittet.

\footnotetext{
${ }^{1}$ Laut gütiger Mitteilung von Herrn Oberbibliothekar P.-D.Dr. STrahm in Bern sind sechs Briefe ScновіNGERs in Bd. III und V der Sammlung «Epistolarum ab eruditis viris ad Alb. Hallerum scriptorum. Latinae. Bern 1773/74» abgedruckt, jedoch mit Ausnahme eines Briefes stark gekürzt.
} 
Erwähnt sei auch Cleophea Schobinger geb. Zollikofer (1638-1692), Gattin des Bartholome Schobinger (1635-1705), Pursner im Notenstein. Diese hatte sich durch eifriges Studium, Sektion von Frauenleichen und praktische Tätigkeit zu einer ausgezeichneten Geburtshelferin ausgebildet. Sie wird in den Briefen von Wepfer, Peyer und Joh. von Muralt erwähnt und letzterer nennt sie «eine göttliche und heilbringende Stütze der Schwergebärenden» (C.Brunner und W.v. Muralt).

Der Vater des Briefschreibers war Johann Burkhard Sghobinger (1701-1743), Lizentiat der Rechte und 1735 Obervogt der Zollikoferschen Herrschaft zu Altenklingen (Thurgau). Seine Frau war Barbara Giller, die Schwester des oben erwähnten Peter Giller. Sein Sohn David Christoph wurde am 30. Januar 1726 in St. Gallen geboren und besuchte nach der Übersiedelung des Vaters nach Altenklingen das Gymnasium in Winterthur, wo er Humaniora und Philosophie studierte. Nach dem Tode des Vaters kehrte er nach St.Gallen zurück und erhielt hier von seinem Onkel GILler anatomischen Unterricht. GILLER stellte ihm auch seine reichhaltige Bibliothek zur Verfügung und gab ihm Anleitung zur Herstellung einfacher Arzneien. Von ihm wurde Schobinger an Halder empfohlen, der ihn 1745 auf der Rückreise von Bern nach Göttingen mitnahm und ihn dort mit väterlicher Fürsorge umgab. Außer Haller, der in Anatomie, Physiologie, Chirurgie und Geschichte der Anatomie unterrichtete, waren dort seine Lehrer: G. Richter ${ }^{2}$ für Materia medica, Pathologie, Semiotica und allgemeine medizinische Praxis, Andreas Segner ${ }^{3}$ für Experimentalphysik und Јон. Gottrried Brendelius' für Chemie, Arzneiverordnungslehre, Materia medica, Semiotica und spezielle Praxis (siehe Curriculum vitae in Schobingers Dissertation).

Zu der Doktorpromotion des Joh. Heinrich Oschwald von Schaffhausen (19. April 1747) verfaßte SchовINGer eine Gratulationsschrift mit dem Titel De ortu bilis cysticae, et ejus ad vesiculam felleam itinere. Auf 19 Seiten werden hier die verschiedenen Ansichten über den Ursprung der Galle in der Gallenblase besprochen. Ihre Herkunft aus den Ductus hepatocystici wird abgelehnt, ebenso die Sekretion durch die Drüsen der Blase selbst. Demgegenüber wird aus Experimenten und Autopsiebefunden geschlossen, daß die Galle durch den Ductus cysticus in die Blase gelange und dort eingedickt werde, entsprechend der von HaLLER geäußerten Auffassung.

${ }^{2}$ Geong Gotrlob Richter (1694-1773), 1736 Professor in Göttingen.

${ }^{3}$ Joh. Andreas Segner (1704-1777), 1733-1755 Professor in Göttingen, nachher in Halle.

${ }^{4}$ Joh. Gottfried Brendel (1712-1758), 1738 Professor in Göttingen. 
Schobingers Dissertation, die 77 Seiten umfaßt, trägt den Titel: De telae cellulosae in fabrica corporis humani dignitate. Es ist dies eine sehr tüchtige und eingehende Arbeit, die in mancher Beziehung ganz modern anmutet. Unter Tela cellulosa versteht der Verfasser das überall im Körper verbreitete Bindegewebe, das teils aus Fasern, teils aus Lamellen besteht. Da Haller beim jungen Foetus an Stelle des Bindegewebes eine schlüpfrige Gallerte fand, wird die Bildung der Fasern aus Flüssigkeiten angenommen, eine Ansicht, die heute wieder vertreten wird und experimentell gestützt is ${ }^{5}$. Gefärbte Flüssigkeiten, in große Arterien injiziert, färben das Bindegewebe, und durch Injektion von Wasser, welches durch die Ostien der Gefäße austritt, läßt sich ein Hydrops nachahmen ${ }^{6}$. Die Flüssigkeit füllt hiebei die Zwischenräume zwischen den Fibrillen aus. Diese Zwischenräume (cellulae) kommunizieren im ganzen Körper, wofür als Beweis das subkutane Emphysem angeführt wird. Scновinger erwähnt hier ferner das Verfahren von Vieh- und Roßhändlern, welche von einer kleinen Wunde aus die Unterhaut aufblasen, um den Tieren ein besseres Aussehen zu verleihen. Auch könne man bei Anasarca durch Anbrennen der Fußsohle bis zum subkutanen Bindegewebe die Flüssigkeit aus dem ganzen Körper entleeren (BoErhaAve).

Eine besondere Art des Bindegewebes ist das Fettgewebe (Tunica adiposa), in welchem das Fett von den Arterien sezerniert werden soll. Das Fett kann durch Wärme oder körperliche Arbeit verflüssigt, von den Venen resorbiert und konsumiert werden, z.B. bei akuten Fiebern. Fettgewebe kann auch die Muskulatur ersetzen. Durch Nadelstiche wird festgestellt, daß der Panniculus adiposus gefühllos ist, weil hier nur wenige Nerven verlaufen. Die Funktion des Fettes wird im Schutz vor Kälte erblickt, wofür als Beweis angegeben wird, daß Körperteile, in welchen das subkutane Fett fehlt oder spärlich ist (Nasenspitze, Finger und Zehen) zuerst der Erfrierungsgangrän verfallen, eine Erklärung, die freilich nicht zutreffend ist. Sehr richtig wird jedoch bemerkt, daß Binde- und Fettgewebe die Verschiebung der Muskeln und der Haut ermöglichen. Ferner soll das Fett durch Ausfüllen der Lücken zur Verschönerung des Körpers beitragen.

${ }^{5}$ Siehe Doljanski und Roulet (Virchows Arch. Bd.291, 1933). In der Gewebskultur wurde die Entstehung kollagener Fasern frei im plasmatischen Medium nachgewiesen.

${ }^{6}$ Schobinger scheint sich hier auf Experimente Hallers am toten Tier zu stützen. Im lebenden Tier läßt sich, wie die klassischen Versuche von Сонмнегм und Liснтнегм (Virchows Arch. Bd. 69, 1877) gezeigt haben, durch künstliche Hydrämie kein echter Hydrops erzeugen. 
Hierauf wird eine sehr genaue, geradezu vorbildliche Beschreibung des Bindegewebes im ganzen Körper gegeben, wobei oft die Methode des Aufblasens verwendet wird. Mit dieser Methode können z. B. die beiden Blätter des Netzes getrennt werden. Bei den Lungen wird angegeben, daß sie nicht aus isolierten Läppchen bestehen, denn diese kommunizieren alle unter sich. Die Bläschen sind nicht geschlossen, die Ampullen nicht blind. «Unde demum justissima conclusio summo viro (HALLER) est enata, cellulosum, non vesiculosum pulmonem esse.» Welchen Bronchus man immer aufbläst, immer geht die Luft durch die ganze Lunge?. Wegen der schnellen Strömung des Blutes wird von den Arterien in der Lunge kein Fett abgelagert.

Im Auge werden der von feinsten Septula (Winslow) durchzogene Glaskörper und die Linse, deren Kapsel sich durch Einblasen von Luft ablösen läßt, auch zur Tela cellulosa gerechnet. Ebenso gehören Sehnen, Bänder, Fascien, Periost und Perichondrium zur Tela cellulosa, denn sie gehen in lockeres Bindegewebe über. Auch die Dura mater und das Integumentum nehmen bei Maceration die Beschaffenheit der Tela cellulosa an. Umgekehrt wird das weiche lockere Gewebe vom fötalen Leben an durch Wasserverlust immer straffer (was an moderne Theorien des Alterns erinnert).

Zwischen Muskelfasern und Cellulosafasern sieht Sсновіnger keinen großen Unterschied. Den ersteren kommt aber als spezifische Eigenschaft die Fähigkeit der Kontraktion und Motion zu.

Wenn man in der Arbeit Schoвingers auch überall die Inspiration HaLLERS spürt, so hat der Verfasser doch das große Verdienst, die bisher vernachlässigte Rolle des Bindegewebes systematisch nach der morphologischen und funktionellen Seite erforscht zu haben. Die Methoden der Inflation und Maceration haben ihm manche Erkenntnisse geliefert, die an die modernen Präparationsergebnisse Benninghoffs und seiner Schule anklingen.

Haller selbst hat zu Schobingers Doktorarbeit ein sehr herzlich gestimmtes Nachwort geschrieben, in welchem er seiner Freude über die erfolgreichen Studien seines Schülers und sein gutes Betragen Ausdruck gibt. Es heißt hier: «Accepi Te, mi Schobingere, ab Avunculo tuo, Amico mio vetere, fido, unico in studiis communibus, cujus amicitiam, quoad vivent mea carmina, scient posteri, inter summa vitae bona me numerasse.

${ }^{7}$ Eigene Versuche an Kalbslungen haben mir gezeigt, daß diese Beobachtung von HaLleR und Sсновinger durchaus richtig ist. Die Konsschen Porenkanäle und die interlobären Parenchymbrücken ermöglichen das Übertreten der Luft von Alveole zu Alveole und von Lappen zu Lappen. 
Meae fidei Te, carissimum nepotem, et ex amoris lege unicum filium, credidit vir optimus. Eum ipsi reddo talem, qualem vix spe aut votis, credo, exspectasset, adeo ab omni vel minima vitae macula immunem, in liberrima studiosorum civitate innocentia, integritate morum, indefesso studio, unico veri et boni amore insignem, ut meos filios Tui similes ultimis meis votis et audacissimis a summo Bonorum Datore efflagitem.» ${ }^{8}$

Am 18. Mai 1748 wurde Schobinger unter dem Vorsitz Hallers in Göttingen zum Doktor promoviert.

SchobIngers Promotion hat übrigens zu weiteren wissenschaftlichen Untersuchungen Anlaß gegeben. G.G.RichTER, sein Lehrer, schickt der Disputation von Schobingers Dissertation eine längere Abhandlung voraus: De duplici novo inflammationum exitu rigescendo et desquamando. Hier wird dargelegt, daß eine Entzündung nicht allein in Eiterung und Gangrän, sondern auch in Verhärtung und Erstarrung (wie bei Gelenkversteifung) oder in Abschuppung (wie bei Masern und Scharlach) ausgehen könne.

Ferner hat Schobingers Freund und Studiengenosse Daniel Langhans ${ }^{9}$ aus Bern ihm eine Arbeit «De causa a pastu oriundae somnolentiae» gewidmet. Zunächst wird hier die Ansicht abgelehnt, daß der Druck des vollen Magens auf die Aorta oder die Erschwerung der Blutzirkulation in den kleinen Hirngefäßen die Schläfrigkeit während der Verdauung erzeuge. Vielmehr ist die Ursache der Somnolenz die Reizung der zahlreichen Magennerven durch die Speisen. Durch den Speisebrei und die freiwerdende Luft wird der Magen erweitert, und während der ganzen Verdauung besteht ein vermehrter "Adfluxus spirituum et sanguinis», wodurch das Gehirn der dem Bauch zuströmenden Menge des Blutes und der Spiritus be-

${ }^{8}$ Frei ins Deutsche übersetzt: «Ich habe Dich, mein Scнoвinger, von Deinem Onkel, meinem alten, treuen und einzigen Studienfreund übernommen, dessen Freundschaft ich, so lange meine Gedichte leben und wie auch die späteren Generationen wissen sollen, zu den höchsten Gütern des Lebens gezählt habe. Meiner Treue hat der vortreffliche Mann Dich, den teuersten Neffen und nach dem Gebot der Liebe einzigen Sohn, anvertraut. Diesen gebe ich ihm so zurück, wie er ihn kaum nach Hoffnung und Wünschen erwartet hätte, nämlich unberührt von dem kleinsten Makel des Lebens, ausgezeichnet durch Unbescholtenheit in der freiesten studentischen Gemeinschaft, Reinheit der Sitten, unermüdlichen Eifer, ausschließliche Liebe zum Guten und Wahren, daß ich in meinen höchsten und kühnsten Wünschen den Geber alles Guten bitte, meine eigenen Söhne möchten Dir ähnlich sein.»

${ }^{9}$ Daniel Langhans (1728-1813) aus Bern studierte und promovierte in Göttingen, praktizierte in Bern und in Frankreich, wurde 1754 vierter und 1779 dritter Stadtarzt von Bern und veröffentlichte mehrere medizinische Abhandlungen (THURNHEER). 
raubt wird. Die «Spiritus agiles» genügen dann nicht mehr zum Wachsein. Zugleich werden die Verdauungssäfte (Galle, Magen-, Darm- und Pancreassaft) reichlich abgesondert.

Die praktische Nutzanwendung dieser Auffassung besteht darin, daß man bei Schlaflosigkeit und Fieberdelirien den Schlaf durch Ableitung des Blutes in die unteren Körperteile herbeiführen könne. Hiezu werden Fußbäder, Friktionen der Füße, Phönigmen (Hautrötungsmittel) und Blasenpflaster empfohlen.

Schobinger kehrte über Straßburg, wo er sich noch ein halbes Jahr zur praktischen Ausbildung aufhielt, nach St. Gallen zurück und eröffnete hier im Frühjahr 1749 eine ärztliche Praxis. 1753 verehelichte er sich mit BARbara Stehelin und nach deren Tod mit Ursula Schobinger (1760). Zwei Kinder aus erster und drei aus zweiter Ehe starben im frühen Kindesalter und nur eine Tochter erreichte das vierzehnte Lebensjahr.

An öffentlichen Ämtern fielen Schовinger folgende zu: 1760 Registrator Bibliothecae, 1768 Eilfer der Zunft zu Schmieden, 1776 Zunftmeister, 1781 Bibliothecarius, 1783 Ratsherr, 1784 Prestenamtsverwalter, 1785 Inspector Bibliothecae et Censor librorum, 1786 Obmann bei den Examina chirurgorum. Im Jahre 1765 wurde er außerdem zum Professor der Philosophie an der höheren Lehranstalt als Nachfolger des an die Ritterakademie in Berlin berufenen JАСов Wegelin ernannt. Diese Lehranstalt war eine kleine theologische Fakultät, 1712 unter der Ägide des Dekans und Professors BarthoLOME WegeLIN (1683-1750) gegründet. In einem 1830 erschienenen Büchlein, welches die Geschichte dieser Lehranstalt behandelt, heißt es über Schoвinger: «Der Verlurst ${ }^{10}$ wurde durch die 1765 zufolge Wägelins Empfehlung auf den Doktor der Arzneikunst David Christopi SchoвinGER gefallene Wahl zwar nicht ganz ersetzt; aber doch war Schoвinger gelehrt, vielseitig und beweglich, und nur in der Bildung und Darlegung eigener Ansichten zu furchtsam.» Hier wird also bestätigt, was GILler in seinen Briefen an Haller über seinen Neffen schreibt, indem er wiederholt seine Schüchternheit und Ängstlichkeit hervorhebt.

Die dreizehn Briefe, welche Schoвinger in den Jahren 1748-1766 an HALLER geschrieben hat, sind in lateinischer Sprache abgefaßt. Sie wahren überall die respektvolle Distanz zwischen dem Schüler und dem berühmten Lehrer, wobei Scновіnger nie müde wird, mit oft überschwenglichen Worten seinem ehemaligen Mentor seinen Dank für die genossene Ausbildung

${ }^{10}$ D.h. der Wegzug von JACoB WegeliN. 
und die ihm in Göttingen erwiesenen Wohltaten zu bezeugen. Stets kehren auch die innigsten Wünsche für Hallers Gesundheit und Wohlergehen wieder. Die Verehrung des Briefschreibers für Haller äußert sich auch in der Anrede, welche in den meisten Briefen lautet: «Vir Illustris, Excellentissime, Experientissime, Patrone, Praeceptor, Euergeta ad cineres usque pio animo colende!» Dementsprechend ist auch die Unterschrift mit Superlativen geschmückt: «Celeberrimi et inclyti Nominis Tui Cultor devotissimus, obstrictissimus D. Chr. SCHobInger.»

In den beiden ersten Briefen ${ }^{11}$, datiert vom 9. November 1748 und 17. März 1749, berichtet SсновіNGer über seine Erlebnisse und Eindrücke in Straßburg (lateinisch Argentoratum). Aus der Reise dorthin sei er oft über den Stand der Göttinger Akademie befragt worden: «mirantibus plurimis novae, ut dicunt, Academiae florentem statum», und stolz setzt er hinzu: «Quae ceterum Goettingae vidi, audivi, hic nunquam vidissem, vel auribus percepissem.» EisenmanN ${ }^{12}$, Rektor der Straßburger Akademie, entlockte ihm ein Lächeln, als er ihm bei seiner Ankunft sagte, er sei gewiß wegen der Menge und Eleganz der anatomischen Demonstrationen nach Straßburg gekommen. Die Zahl der Leichen genügt freilich, aber die Präparate werden schon vierzehn Tage vor der Demonstration hergestellt und schrumpfen und verderben deshalb. Besser gefällt Schobinger der Unterricht in der Chirurgie. Dr. Le Riche, der am Lebenden und an der Leiche voroperiert, gestattet den Hörern, die Operation ein- oder zweimal an der Leiche nachzumachen. Vormittags besucht Schoвinger das französische Krankenhaus (Nosodochium gallicum), wo die chirurgisch Kranken sorgfältig gepflegt und behandelt werden. Von Dr. LINDERN ${ }^{13}$ wird gesagt, daß er beinahe alle seine Pflanzen in natürlichen Farben malen lasse.

In seinem zweiten Brief weist Schobinger dem Chirurgen Le Riche einige grobe Fehler nach. So habe er vergessen, daß man bei einem Aneurysma der Art. brachialis die Arterie wegen der Collateraläste doppelt unterbinden müsse. Er habe bei der Operation einer Tränenfistel eine falsche Schnittführung angewandt, und bei einem hirnverletzten Soldaten, bei welchem nach Schoвingers Meinung durch Trepanation und Aderlaß noch etwas zu erreichen gewesen wäre, habe er wegen gleichzeitiger Kontusion

${ }^{11}$ Hallersche Briefsammlung, Bd. 7, Nr.98, und Bd.8, Nr. 25.

12 Georg Heinrich Eisenmann (1693-1768), Arzt und seit 1733 Professor für Physik und Pathologie in Straßburg, Herausgeber von Tabulae anatomicae IV uteri duplicis.

${ }^{13}$ JosePh LiNDERn (1682-1755), Arzt und Botaniker in Straßburg. 
des Auges Umschläge (fomenta) verordnet, worauf bald nachher der Tod an Gangrän erfolgt sei. «Talia peragunt quotidie et pejora.» In diesem letzteren Fall muß man sich freilich fragen, ob nicht der erfahrene Le Riche mit seiner konservativen Therapie die Prognose richtiger gestellt hat als der junge Schobinger.

In den höchsten Tönen preist dieser den geburtshülflichen Unterricht des Dr. Fried ${ }^{14}$, der sich durch Klarheit und Sorgfalt auszeichnet. Sehr viel leistet hier der künstliche Uterus mit Foetus, der zur Demonstration der Encheiresen gebraucht wird. «Instrumentis paucis et rarissime utitur. Forcipes fere omnes fugit.» Denn wegen des Einführens der Zange bei engen Geburtswegen entstehen schwerste Kontusionen, nur bei freiem Kopf ist die Zange erlaubt. Aber wenn die eine Schulter am Sacrum, die andere am Os pubis fixiert und der untere Arm gelöst (emissus) ist, dann ist die Anwendung der Zange gestattet ${ }^{15}$. Haken werden sehr selten gebraucht. Fried ist in allem sehr wahrheitsliebend, im übrigen ein «orthodoxer» Arzt, allen anderen Straßburger Ärzten vorzuziehen und der Verehrung und Liebe seiner Schüler wert. $\mathrm{Zu}$ bedauern ist, $\mathrm{da} ß$ er wegen seiner großen Praxis nicht mehr Schriften herausgeben kann. Seine Dissertation, De vulnere gravidi uteri sclopetario (Schußverletzung) feliciter post puerperium curato, ist leider nicht mehr aufzutreiben. Im übrigen sucht Scновinger für HaLler Dissertationen zu sammeln, hat aber damit nur wenig Erfolg. Er nennt eine Arbeit von MaUchart ${ }^{16}$, De pulsu crepitando cordis in maximo aortae aneurysmate, und eine des Berners Nöttinger ${ }^{17}$, De Arteriotomia. Endlich dankt er Haller dafür, daß dieser in seinem Streit mit HamBERGER $^{18}$ in Jena auch ihn (Sсн.) als Zeugen angeführt habe. Ein Schüler Hambergers habe die in Jena gehörte falsche Theorie in Straßburg noch

14 Јон. Јаков FriEd (1681-1769) gründete 1728 die Straßburger Gebäranstalt und Hebammenschule und war als Lehrer berühmt.

${ }^{15}$ Diese Vorschrift entspricht durchaus den jetzigen geburtshülflichen Regeln (gütige Mitteilung von Dr. Käser, Chefarzt der Frauenklinik St. Gallen).

16 Burchard Daniel Mauchart (1696-1751), 1726 Professor für Anatomie und Chirurgie in Tübingen.

${ }^{17}$ Samuel Friedrich Nöttingen aus Bern promovierte am 29. April 1747 in Straßburg mit einer Arbeit De arteriotomia, ejus recto usu et injusto neglectu.

${ }^{18}$ Georg Erhard Hamberger (1697-1755), Professor in Jena. Mit ihm führte Haller einen heftigen wissenschaftlichen Streit wegen der Theorie der Atmung. Hamberger nahm an, daß sich Luft im Pleuraraum befinde und daß die Intercostalmuskeln die Rippen herunterziehen. 
fälscher vorgetragen, «ut horror audienti natus sit». Professor BoEcLen ${ }^{19}$ jun. habe ihn jedoch «acerrima bile» bekämpft.

Alle folgenden Briefe ${ }^{20}$ sind von St. Gallen aus geschrieben. Sсновіnger erzählt, wie seine Praxis allmälig in Gang kommt und wie er dabei von seinem Onkel Giller unterstützt wird. Freilich beklagt er sich hie und da über seine Mitbürger, welche mehr Zutrauen zu den Veteranen des Ärztestandes als zu einem jungen Arzt haben, der sich an die einfache Wahrheit und nicht an die bei Weibern beliebten abergläubischen Vorspiegelungen hält. Diese Schwierigkeiten und die geringen Einkünfte stimmen ihn manchmal melancholisch, «non certe ex avaritiae principio, sed ideo, quod nihil magis in votis esset, quam artem in utilitatem aliorum et Dei gloriam imprimis quam curatissime et diligentissime exercere». In solchen sorgenvollen Stunden schöpft er neuen Mut im Gedenken an Hallers Wohlwollen: «Tunc et animum spes laetior adfulsit et omnia fortius ferre pro tempore coepi.» Im Jahre 1755 hält sich seine Praxis immer noch in engen Grenzen, aber er darf doch ohne Ruhmredigkeit (sine jactantia) sagen, daß er viele glückliche Kuren gemacht und begangene Fehler auch bei älteren erfahrenen Kollegen gesehen hat. Wenn er alten Vorurteilen und den Ratschlägen der Weiblein (muliercula) folgen und sich der Scharlatanerie hingeben würde, so hätte er mehr zu tun. Als Freund hat er Dr. Zollikofer ${ }^{21}$ gewonnen, den HaLLER seinerzeit in Halle bei Coschwitz ${ }^{22}$ getroffen hat.

Seine freie Zeit füllt Schoвinger mit Lektüre und Einreihung und Gruppierung seiner Beobachtungen aus. Er bedauert, daß er wegen der Kosten von der Anschaffung größerer Bücher und von wissenschaftlichen Versuchen (a variis experimentunculis, ne dicam experimentis) absehen müsse. Immerhin berichtet er 1756 (Bd. 15, Nr. 41), daß er in Fortsetzung seiner Dissertation Versuche an verschiedenen tierischen Membranen (tunicis nerveis in spongias cellulosas mutandis) angestellt habe. Die Umwandlung einer papierdünnen ausgetrockneten Rinderblase in ein zelliges Gewebe von einigen Linien Dicke sei ihm nach anfänglichen Mißerfolgen schließlich durch Maceration gelungen.

1764 schreibt Schoвinger, daß er Tag und Nacht seine Praxis betreibe, daß er aber seine körperliche Schwäche nicht, wie er es wünschen würde,

${ }^{19}$ Philipp Heinrich Boecler jun. (1718-1759), Professor der Medizin in Straßburg.

${ }^{20}$ Hallersche Briefsammlung Bd. 8, Nr. 40, 85; Bd. 12, Nr. 19; Bd. 14, Nr. 187; Bd. 15, Nr. 41 ; Bd. 23, Nr. 30, 59, 63, 85, 121; Bd. 25, Nr. 6.

${ }^{21}$ Hector Zollikofer (1706-1780), Stadtarzt in St. Gallen.

${ }^{22}$ Professor Coschwitz, Anatom in Halle, schon in Gillers Briefen erwähnt. 
ausschalten könne. Er habe einige Beobachtungen gesammelt, welche er gerne den Acta helvetica in Basel zur Publikation schicken würde. Sie seien aber noch nicht ausgearbeitet und er wisse nicht, ob sie des Druckes würdig seien (Bd. 23, Nr. 30).

Am 7. Februar 1764 zeigt Schobinger den Tod seines Onkels Giller an, der unerwartet an einer Apoplexie gestorben sei. "Intra bihorium vivis ereptus ad beatos coelites transiit.» Man betrauert in St. Gallen einen klugen Magistraten - GiLler war zuletzt Unterbürgermeister - und einen sehr erfolgreichen (felicissimum) Arzt. Schobinger hat die letzten Briefe (literas humanissimas), die Haller an Giller gerichtet hat, gelesen und er bemerkt dazu: «Cum dulcissimo animi gaudio amicitiae ejus in animo tuo nihil tot annorum spatio decessisse cognovi.» Für die dem Onkel erwiesene Freundschaft gebührt Haller der wärmste Dank (Bd. 23, Nr. 30).

Im gleichen Jahr meldet Schовinger, daß Gott ihm einen schönen und lebhaften Knaben geschenkt habe (Bd.23, Nr.121). Es sei nun seine größte Sorge, ihn am Leben zu erhalten. «Pulles farinaceae (Mehlbrei) o si per omnem Europam damnarentur publice, tot infantulis funestae.» Der Mehlbrei wird also verpönt, eine sehr richtige Erkenntnis und Forderung, welche die Pädiatrie erst in unserem Jahrhundert völlig durchgesetzt hat. Leider verlor Schobinger auch dieses Kind schon im zartesten Alter.

Zu seiner Ernennung zum Professor der Philosophie und lateinischen Sprache an der höheren Lehranstalt (1765) bemerkt Schoвinger, daß er dieses Amt um so lieber angenommen habe, als es die Ausübung der Medizin nicht hindere. Wenn manche Mitbürger es bereuen, daß ihm vor zwei Jahren das Amt des Archiaters abgeschlagen worden sei und sie diese Sache in Wiedererwägung ziehen wollten, so erfreue er sich jetzt einer wesentlich höheren Besoldung, als sie die St. Galler Stadtärzte erhalten (Bd. 25, Nr.6).

In medizinischer Hinsicht erfahren wir aus SchoвINGERs Briefen manches Interessante. Zunächstbeschäftigtihn die Krankheit seines Onkels GILlen ${ }^{23}$, welcher im Herbst 1749 von einem Fieber ergriffen wurde, das weniger wegen seiner Heftigkeit als durch seine Begleiterscheinungen dem Patienten gefährlich wurde. Beim Aufrichten des Kranken bekam dieser Ohnmachtsanfälle mit konvulsivischen Bewegungen, Aphonie und Artikulationsstörungen, so daß man bei höchstem Kräfteverfall das Ende befürchten mußte. Das Blut gelangte kaum mehr in den Kopf, und der Kranke hörte

${ }^{23}$ Giller litt in den Jahren 1734-37 wahrscheinlich an einer Lungentuberkulose (siehe meine frühere Publikation). 
die Arterienpulse im Kopf wie ein Rauschen des Windes («adeo, ut ille singulos arteriarum pulsus intra caput tanquam venti strepitum audiret»). Es wurde also die cerebrale, orthostatisch bedingte Anämie richtig diagnostiziert. Von einem bestimmten Tag an trat dann eine Besserung im Befinden des Patienten ein. Merkwürdig ist, daß Schobınger die Krankheit hauptsächlich auf einen nur zum Teil abgetriebenen Bandwurm (Taenia) zurückführt. Wegen GiLlens großer Schwäche habe er keine Bandwurmmittel (Anthelminthica), sondern nur Antifebrilia und Analeptica verordnen können. Sobald es dem Patienten besser gehe, werde er ihm Vinum sedlicense ${ }^{24}$ verabreichen. Ferner bittet er den in Göttingen weilenden Arzt J.G.Zrmmermann ${ }^{25}$, ihm seine Dissertation über die Taenien zu schicken, und bemerkt dabei, daß diese Bandwürmer in St. Gallen nicht selten seien (Bd. 8, Nr. 85).

1753 war GiLler wieder oft ans Bett gefesselt, er werde von heftigen Schmerzen in der Magengegend gequält, und Schobinger meint, er leide an Obstruktion der Abdominalgefäße, vor allem der Pfortader. Auch seien Hämorrhoiden vorhanden, aber Blutegel und ein Aderlaß am Fuß haben den Plethoriker nur wenig erleichtert (Bd.12, Nr.19).

Sodann beschreibt Scновinger mehrere eigentümliche Krankheitsfälle, welche für seinen einstigen Lehrer Interesse haben könnten, nämlich:

1. Ein gewaltiges Sarkom bei einem 6-7jährigen Knaben, das die ganze Augenhöhle ausfüllt, mit den Lidern verwachsen ist und über das Gesicht herunterhängt. Es sei überall rot und vaskulös, und weil es die Tränenabführwege verlege, sammle sich mit Eiter vermischtes Sekret an. In der Tiefe der Geschwulst, die von der Nase und der Gegend hinter den oberen Schneidezähnen ausgegangen sei, lasse sich der Augapfel fühlen. «Ancipiter valde haeremus omnes, quid agendum sit?» SCHовINGER bedauert, daß in St. Gallen kein Chirurg sei, der heroische Operationen schnell und sicher ausführen könnte. Er selbst meint, daß man die Geschwulst vorsichtig exzidieren könnte, um das schreckliche Aussehen des Gesichtes zu beseitigen, das Auge sei ohnehin verloren. Aber Giller will der Operation nicht zustimmen (Bd. 8, Nr.40).

2. Hepatitis ohne Icterus, deren Vorkommen von BoERHAAVE negiert wurde. «Hepatitis adeo subinde sine ictero, quam ursit Ill. BREndeLIus, vera est. Forte inflammatio in solis hepatis arteriis sine dubio haesit. ${ }^{26}$ Der Fall ging in Heilung aus unter Anwendung von Antiphlogistica und V. sonibus (?) (Bd.8, Nr. 85).

${ }^{24}$ Wahrscheinlich ein bittersalzhaltiges Abführmittel. Bekannt ist Aqua sedlicensis von Sedlitz (Böhmen).

${ }^{25}$ Јон. Georg Zimmermann (1728-1795) von Brugg, berühmter Arzt, Biograph Hallers, später Leibarzt des Königs von England in Hannover.

${ }^{26}$ Hepatitis ohne Gelbsucht kommt nach unseren heutigen Kenntnissen sicher vor, nur trifft die SсновINGERsche Erklärung (Entzündung der Arterien) nicht zu. 
3. Im Jahre 1749 war ein preußischer Augenarzt namens Cyrus in St. Gallen, in Theorie und Praxis und besonders in der Anatomie des Auges wohl erfahren. Er hat mehrere Fälle von grauem Star zu billigem Preis kuriert. Seine Operationsmethode weicht von der bisherigen in mehreren Punkten ab. Erstens sticht er die Nadel nicht im äußeren unteren, sondern genau im äußeren mittleren Teil der Albuginea und Sclerotica zwei Linien von der Cornea entfernt ein. Zweitens verwendet er nicht eine lanzenförmige oder abgerundete, sondern eine überall runde Nadel mit feinster Spitze, und drittens braucht er kein Augenspeculum, sondern er hält das Auge mit Daumen und Zeigefinger der linken Hand fest. «Felix nostro loco fuit» (Bd. 8, Nr. 85).

4. Da Haller 1749 eine Arbeit «De morbis ventriculi» (4. Programma Goettingae) geschrieben und darin auch über die Ulcera ventriculi berichtet hat, beschreibt SсновINGER die Krankengeschichte und den Sektionsbefund eines Mannes, der in der vorderen Magenwand ein großes Loch vom Durchmesser eines sanktgallischen 15-Kreuzer-Stückes $(2,8 \mathrm{~cm})$ aufwies, durch welches der gesamte Mageninhalt in die Bauchhöhle ausgetreten war. Die Autopsie wurde von Dr. WILD ${ }^{27}$ ausgeführt (Bd.14, Nr.187).

5. Mit Giller hat Schoвinger einen Fall von akuter Arsenvergiftung beobachtet. Es handelte sich um einen jungen Mann, der Mäusegift verschluckt hatte. «Er aß Maußzeltlein, ut hic vocant.» Der Magen zeigte bei der Autopsie um die Cardia und an den meisten Stellen eine sehr schöne Injektion (pulcherrimam injectionem anatomicam) und enthielt sehr viel rötlichen Schleim, wie er schon während des Lebens erbrochen worden war. Die Därme waren unversehrt. Geruch nach Knoblauch fehlte, aber auch jeglicher Fäulnisgeruch, obwohl die Sektion erst 27 Stunden nach dem Tode bei heißem Wetter stattfand. Die Leiche war auch nicht stark aufgetrieben, und es waren sozusagen keine Blutkoagula vorhanden (Bd.14, Nr. 187).

Hier wird wohl über eine der ersten Leichenöffnungen bei akuter Arsenvergiftung berichtet, lange bevor GroHe und MosLer im Jahre 1865 in Virchows Archiv Bd. 34 ihre angeblich erste Autopsie eines solchen Falles publizierten. Es zeugt von ScновINGERS trefflicher Beobachtungsgabe, daß er den fäulnishemmenden Einfluß des Arsens klar erkannte. Wenn er auf das Fehlen des Knoblauchgeruches hinweist, so ist seither bestätigt worden, daß dieser Geruch durchaus kein konstantes Merkmal der Arsenvergiftung ist.

6. Senkungsabsze $\beta$ bei einer 23jährigen Frau, der sich von der Lendengegend nahe dem Dickdarm abwärts zwischen den Muskeln des Gesäßes und Oberschenkels ausbreitete.

7. Knorpelharter Scirrhus des Pylorus, übergreifend auf Pancreas und Duodenum, mit einem Loch in die Höhle des Mesocolon.

8. Bei einem Hämorrhoidarier mit Darmprolaps entleerte sich Eiter aus dem Ende des Darmes. Da weder ein Geschwür noch eine Fistel vorhanden war, ist SchoвINGER überzeugt, daß der Eiter aus den entzündeten Gefäßen des Prolapses stammte («hoc pus ex vasis inflammatis transsudare sine substantiae laesione»).

9. Die linke Lunge eines Hydropikers und Phthisikers, der von Dr. Zollikofer seziert wurde, fand sich voll Eiter «sine substantiae ulla consumtione». Die Lunge wurde überall eingeschnitten, aber es war keine Höhle vorhanden, sondern überall solides zellulöses Ge-

${ }^{27}$ Dr.med.EsaJAS WiLd (1722-1758), Arzt in St. Gallen. 
webe, das bei Druck sehr viel Eiter entleerte. Bronchien und Gefäße waren intakt. Wahrscheinlich handelte es sich hier um multiple kleinste Abszesse oder um eine eitrige Bronchiolitis.

10. Bei einer robusten Magd mit unregelmäßigen oder aussetzenden Menses sah ScHoBINGER entzündliche Schwellungen an den Fingern mit nachfolgenden Rissen der Haut, welche zur Zeit der ausbleibenden Menses oder der sehr spärlichen Blutung auftraten. Es wird sich hier um ein vasoneurotisches Menstruationserythem gehandelt haben.

11. Ein neugeborenes Kind, welchem die Hebamme das Frenulum der Zunge zu stark gelöst hatte, starb an der Retraktion der Zunge in den Rachen. Eine Blutung war nicht vorhanden.

12. Bei einem Mann mit einer großen Quetschrißwunde am Hinterhaupt hing die ganze Haut mit der Galea und den Musculi occipitales herunter, so daß fast nur das weiße Pericranium übrig blieb. Beim Auswaschen der Wunde mit wäßrig verdünntem Essig war dieser Teil gefühllos, während der herabhängende Hautlappen sehr sensibel war.

Die Beobachtungen (observatiuncula) 6-12 sind in Bd. 23, Nr. 30 der Briefsammlung enthalten.

13. Im Jahre 1764 berichtet SchовINGER, daß er einen Fall von schwerer Hypochondrie außer durch leichte Evacuantia und Roborantia allein durch Massage des Bauches («abdominis frictionibus deorsum factis») geheilt habe. Er habe einen Rückfall befürchtet, und Haller wisse wohl aus eigener Erfahrung, wie schwierig solche Patienten seien, wenn sie sich krank fühlen, geschweige denn, wenn sie sich gesund glauben. Aber seit einem Jahr schweige das Übel vollkommen in dem Kranken (Bd. 23, Nr.63 und 85).

Man ersieht aus diesen Mitteilungen, die ich hier stark gekürzt wiedergegeben habe, daß Sсновіnger ein guter Beobachter und sorgfältiger Arzt war, der die Besonderheiten der nicht alltäglichen Fälle klar erkannte. Zur Publikation scheint er sich freilich nicht entschlossen zu haben, denn in den Acta helvetica physico-mathematico-botanico-medica Vol. 1-8 (1751-1777) und den Nova acta helv. Vol. 1 (1787) ist keine Arbeit von ihm zu finden. Ob Haller ihn nicht dazu ermuntert hat?

Hallers Unterricht in der Botanik hat auch in seinem Schüler das Interesse für diese Wissenschaft geweckt, denn bald nach seiner Ankunft in St.Gallen schreibt Schoвinger, er habe die benachbarten Wiesen und Waldschluchten (saltus) durchstreift, habe aber nichts besonderes gefunden. Nächstens werde er die Appenzellerberge absuchen, um Hallers Opus stirp. helv. zu bereichern. Vorläufig sammle er Gramineen, die er später bestimmen wolle. «Id certi habeo, me nunquam plantarum amabilissimum sepositurum studium.» Dann folgen noch Angaben über Valeriana alpina mit Blättern der Scrophularia, ferner über Viburnum, Vinca usw.

Ähnlich wie Giller ist auch Schobinger bestrebt, sich eine wissenschaftliche Bibliothek anzulegen, wobei er besonderen Wert auf Hallers 
Werke legt. 1749 erwartet er mit Ungeduld die Zeit, da HaLlers «Methode, die Medizin zu lehren» ${ }^{28}$ und seine Opuscula minora botanica ${ }^{29}$ in den Zürcher Katalogen angekündigt werden, und er hofft auch auf ein baldiges Erscheinen von Hallers neuer Ausgabe der BoerhaAveschen Kommentare ${ }^{30}$. «Tarda nimis talia ad nos perveniunt» (Bd. 8, Nr. 40). Auch HaLLERS Streit mit Buffon (Vorrede zum 2. Band der Buffonischen Naturgeschichte, Leipzig 1752) findet lebhaftes Interesse. «An respondebit unquam objectionibus tuis Buffonius? Vix valebit.»

1764 schreibt Schobinger, daß Giller und er das Studium der Anatomie und Physiologie nie aufgegeben haben. Sie besitzen die ersten fünf Bände von Hallens Physiologie ${ }^{31}$ und erwarten begierig die weiteren drei Bände. Sie haben auch Hallers Abhandlung De irritabilitate ${ }^{32}$ mit Vergnügen und Gewinn (cum voluptate et fructu) gelesen, ebenso die Arbeiten De sanguinis motu (Lausanne 1756), De ovo incubato ${ }^{33}$ und die kleineren Schriften. Das Auctarium stirp. helv. (6 Bände, Basel und Bern 1760-1765) ist noch nicht angekommen (Bd. 23, Nr. 30).

Im gleichen Jahr muß ein Manuskript von HaLlers Physiologie durch die Nachlässigkeit des Buchdruckers verloren gegangen sein, was SсновіNGER aufs tiefste bedauert. «Det Deus novas vires, novam largiatur alacritatem mentis, ut refarcire liceat egregium opus. Verissimum est, hunc nuntium acceptum per aliquot dies omnium rerum mihi creasse taedium, adeo opus hoc amo et desidero.» Die Nachricht vom Verlust dieses Manuskriptes hat ihm also für einige Tage einen wahren Ekel vor allen Dingen eingeflößt (Bd.23, Nr.85). Zwei Monate später aber vernimmt er «cum maximo gaudio», daß das verlorene Manuskript wieder gefunden worden ist. Er gratuliert HALLer zur Rettung des kostbaren Werkes und fügt

${ }^{28}$ H. Boerhaave Praelectiones de methodo studii medici cum peramplis commentariis, Amsterdam 1750.

${ }^{29}$ Opuscula Botanica recusa et aucta, Göttingen 1749.

${ }^{30}$ Hermanni Boerhaave Praelectiones academicae in suas Institutiones rei medicae, cum Comment. Mit Zusätzen zuerst in Göttingen 1740 erschienen.

${ }^{31}$ Elementa Physiologiae corporis humani, 8 Vol. Lausanne 1757-1766.

${ }^{32}$ De partibus corporis humani irritabilibus. Comment. Soc. Reg. Scient. Göttingen 1753. Mémoires sur la nature sensible et irritable des parties du corps animal, 3 Vol. Lausanne 1756-1760.

${ }^{33}$ Eine Arbeit HaLlers mit dem Titel De ovo incubato habe ich weder in seiner Bibliotheca anatomica noch in den Katalogen der Gillerschen und Schoвingerschen Bibliothek auffinden können. 
hinzu: «Quidquid sit, Perillustris vir, ego meo loco aeternas Tibi debeo gratias pro plenissima et optima Physiologia» (Bd. 23, Nr.121).

Im Jahre 1766 fehlt nur noch der 8. Band der Physiologie, der ebenfalls mit Spannung erwartet wird. Hingegen hat Schobinger soeben die neue Ausgabe der Primae lineae physiologiae (Göttingen 1765) erhalten und dankt für dieses fein ausgefeilte Kompendium (Bd.25, Nr.6).

Sehr viel Mühe und Verdruß hat Schoвinger mit der nachgelassenen Bibliothek Gillers, die von dessen Schwiegersohn Hector ZüBlin zum Verkauf ausgeschrieben wurde. Von dieser Bibliothek, die 955 medizinische und naturwissenschaftliche Werke und ungefähr ebenso viele aus anderen Wissensgebieten umfaßte, wurde ein Katalog erstellt, jedoch ohne alphabetische Anordnung und ohne Anmerkung des Preises. Schobinger meldet im Februar 1764, daß er einen kleinen Teil der Bücher gekauft habe und dazu ein sehr schönes Skelett, welches Giller gehörte, sowie das «BlacWELLsche Herbarium ${ }^{34}$ zum Preis von 50 Gulden. Da er schon lange nicht mehr gehofft habe, die medizinischen Werke aus Gillers Bibliothek dereinst zu besitzen, so habe er die wichtigsten Bücher allmälig für sich anderwärts gekauft und habe so im vergangenen Sommer (1763) Hallers anatomische Werke und des Albinus Ausgabe der Eustachiusschen Werke erworben (Bd.23, Nr.30).

Zwei Monate später schickt Schobinger Halder den Katalog, in welchem er die bereits verkauften Bücher gestrichen hat. Gerne hätte er den Preis der einzelnen Werke beigefügt, aber die Erben haben sich dem widersetzt, und Haller soll nun den ihm richtig scheinenden Preis der Bücher nennen, die er für sich erwerben will. Oder er kann, wenn es ihm nicht zu lange dauert, den Preis von den Erben erfahren und dann sein festes Angebot machen. Alle Bücher sind in gutem Zustand (Bd.23, Nr.59).

Haller muß sehr rasch geantwortet haben, denn schon nach neun Tagen schreibt Schobinger, er sei sehr befriedigt, daß er alle von Haller bezeichneten Bücher für ihn reservieren konnte. Er könne sie zu den von den Erben angegebenen Preisen haben. Die Erben hoffen, daß Haller an Stelle der Berner Münzen, welche in St.Gallen im Kurse niedrig stehen, neue Louisdor schicken werde. Die Differenz zwischen Hallers Summe und der Forderung der Erben würde ungefähr einen halben Gulden ausmachen. Die Bücher werden nun gut verpackt an HaLler abgehen, aber SchoвinGER hat die Liquidation der Bibliothek gründlich satt. «Optarem valde, ut

${ }^{34}$ Elisabeth Blackweld, Herbarium selectum emendatum et auctum, 6 Vol. Nürnberg 1750. 
omnia jam in omnem divendita esset mundum, adeo negotiis hujus Bibliothecae torqueor» (Bd.23, Nr. 63).

Mitte Juni 1764 meldet Schobinger den Empfang der Louisdor, die Herr Zeerleder geschickt hat. Letztere hat er dem Schwiegersohn GiLLERS übergeben, der freilich gewünscht hätte, HaLler hätte noch mehr Bücher bezogen. Von den kleineren sei noch eine große Menge vorhanden. Schobinger selbst hat für 12 Gulden die Hallersche Sammlung von chirurgischen Dissertationen gekauft und hat sich gefreut, daß hier die seltensten Fälle chirurgisch und medizinisch aufgeklärt werden (Bd.23, Nr.85).

Die letzte Mitteilung in der Sache der GILlerschen Bibliothek stammt vom 25. August 1764, als Haller Salinendirektor in Roche war. SсновinGER hat den Druck des Kataloges abgewartet, in welchem nun die sehr bescheidenen Preise vermerkt sind. Der Apotheker Struve in Lausanne, welcher von seinem Freunde Züblin, dem Erben Gillers, mehrere Exemplare des Katalogs erhalten hat, ist beauftragt worden, HALLer einen Kata$\log$ kostenlos zu übermitteln. Die Manuskripte sind darin auch verzeichnet, sie sind teils gut, teils schlecht. Diejenigen der Vorlesungen von Albivus, BoerhaAve und $\mathrm{RAU}^{35}$ sind ziemlich gut und schön geschrieben. Wenn etwas davon Haller gefällt, so erwartet Schobinger seine Aufträge. Die Preise soll er selbst bestimmen, der Verkäufer wird einige Abzüge hinnehmen müssen. Der Chorherr Joh. Gessner in Zürich, den Scновinger zu seinen Gönnern zählen darf, hat die physikalischen und medizinischen Dissertationen, im ganzen 82 dicke Bände, zu dem sehr günstigen Preis von 46 Gulden gekauft. «Egregias vero multas possedit beatus Gillerus» (Bd. 23, Nr.121). Aus allen diesen Bemerkungen geht hervor, mit welchem Sammeleifer und Kostenaufwand GiLLER seine Bibliothek geäufnet hatte.

Von seinem Göttinger Studiengenossen Daniel Langhans schreibt Schoвinger, daß dieser ihm eine ausführliche Beschreibung von der Krankheit geschickt habe, welche im Spätsommer 1752 im Simmental grassierte (morbi glanduloso generi adeo infecti ${ }^{36}$ ). Er habe ScновINGER gebeten, diesen Bericht bei nächster Gelegenheit an HaLler weiterzuleiten. Da aber

${ }^{35}$ Giller hatte seinerzeit von den Vorlesungen von Albinus, Boerhaave und RaU (Joh. ЈАКов RAU, 1668-1719) in Leiden Manuskripte angefertigt.

${ }^{36}$ ThurnheEr meint, daß es sich um eine Diphtherie-Epidemie gehandelt habe, wofür die weißen Membranen im Rachen und in der Speiseröhre und die großen Drüsenschwellungen am Hals sprechen. Auffallend ist aber das Auftreten großer Blasen («Blatern») in der Mundhöhle und an verschiedenen Stellen der Haut. 
Langhans sehr dickes Papier (crassissima charta) mit seiner großen Schrift (ampla scriptura) bedeckt hat, so zieht Schobinger es vor, die Langhanssche Darstellung ganz genau (acuratissime et scrupulosissime) auf gedrängtem Raum abzuschreiben, um sie in deutlicher Schrift Hallers Augen zu unterbreiten und zugleich Kosten zu sparen (Bd.12, Nr.19).

$\mathrm{Daß}$ man in St.Gallen streng auf die ärztliche Standesehre hielt und den Mißbrauch ausländischer Titel nicht duldete, geht aus einem Briefe des Jahres 1764 hervor (Bd.23, Nr.59). In St.Gallen habe ein junger Mann namens WARTMANN ${ }^{37}$ angegeben, er sei in Montpellier zum Doktor promoviert worden, er verfüge aber weder über eine verteidigte Dissertation, noch, wie es bei den Franzosen Sitte sei, über eine gedruckte These, noch über ein öffentliches Diplom. Er schütze vor, daß dies in Montpellier nicht gebräuchlich sei, weshalb man mit Recht an seinem Doktorat zweifle. Der Mann komme aus der Chirurgie, er stürze sich aber sofort mit unreinen Händen auf die Medizin (in medicinam subito illotisque involavit manibus).

Nun bittet Schobinger Haller, er möge sich bei Prof. Dr. Sauvages DE LA CRoIX $^{38}$ in Montpellier erkundigen, ob Wartmann den Doktortitel rechtmäßig erworben habe, denn dieser rühme sich, Schüler des Professors Sauvages zu sein. Je nach dessen Antwort werde sich das St. Galler Collegium verhalten.

Im nächsten Brief (Bd.23, Nr.63) dankt Schobinger für die erhaltene Auskunft. WARtManv hat nun sein Doktordiplom von Montpellier zugeschickt bekommen, er hat sich aber keiner Disputation unterzogen. Man preist ihn als gründlichen Chirurgen.

Seinen letzten Brief (Bd.25, Nr.6) läßt Schoвinger durch einen Herrn ERB überbringen, einen frommen und gelehrten Mann, der sich durch seine guten Sitten und seine liebenswürdige Offenheit in St. Gallen allgemein beliebt gemacht hat und nun nach Bern reist (1766). Dieser ist Pfälzer, Seelsorger der armen Gemeinde Rohrbach bei Heidelberg, und hat die Aufgabe übernommen, für den Bau eines Pfarrhauses Geld zu sammeln. Er verdient um so mehr Vertrauen, als er die Kosten seiner Reise selbst trägt und von der gesammelten Summe nichts abzieht. Er wird Haller Grüße von seinem

${ }^{37}$ Bernhard Wartmann (1739-1815), Med. Dr., 1781 Examinator der Wundärzte und Bader, 1789 Ratsherr und Salzdirektor.

${ }^{38}$ Françors Boissier Sauvages de la Croix (1706-1767), Professor der Botanik in Montpellier. 
Arzt Professor Gattenhof ${ }^{39}$ in Heidelberg überbringen. Schobinger bittet HALler, den Herrn Erb mit seiner Autorität in Bern zu unterstützen.

Daß Schobinger an Hallers persönlichen Schicksalen warmen Anteil nimmt, ist selbstverständlich. So beglückwünscht er ihn zu dem vom König von England verliehenen Adelstitel (1749) und zu seinen vermehrten Einkünften. Er kondoliert ihm zum Tod des Söhnchens Albert (1752) und gratuliert zu den Fortschritten von Hallers ältestem Sohn GotTlieb Emanuel und zur Vermählung seiner Tochter mit Fr. Ludwig Jenner. Er ist aufs höchste erfreut über Hallers Rückkehr nach Bern, «ubi Deus Te, Perillustris vir, communi patriae diutissime salvum incolumemque praestet». Mit Bedauern hat er von dem Unfall gehört, bei welchem HaLle R den Arm gebrochen hat ${ }^{40}$. «Dolui suo tempore acerbe infelicem casum fractibrachii.»

1764 begrüßt Schobinger Hallers Rückkehr aus Roche nach Bern: «Ut per Dei Bonitatem in Patria Magnates vestri Te conservare possint, at utique non Tuo in damno, opto» (Bd. 23, Nr. 121). Und zwei Jahre später, als HALLER an eine erneute Übersiedelung nach Göttingen dachte, schreibt Schobinger: Wenn es Haller beschieden sein sollte, nach Göttingen zurückzukehren, so bedaure er dies in Hinsicht auf sein Vaterland, er würde sich aber freuen, wenn HALLER am Orte seiner ehemaligen Wirksamkeit ein wahres, dauerhaftes und seiner würdiges Glück genießen könnte (Bd. 25, Nr.6).

Mit diesem Wunsch am Schlusse seines letzten Briefes bricht SсновıNgers Korrespondenz ab. Wie HaLLer diese aufgenommen hat, wissen wir nicht, denn seine Briefe sind wie diejenigen an GILLER verloren oder an unbekanntem Orte aufbewahrt. Aber Haller muß die Wertschätzung, die er für seinen Göttinger Schüler empfand und die in seinem Epilog zu SсноBINGERs Dissertation zum Ausdruck kommt (siehe S.219), doch beibehalten haben, sonst hätte er nicht auf seine Briefe geantwortet und ihm nicht seine kleineren Publikationen zugeschickt, wie aus Schoвingers Äußerungen eindeutig hervorgeht. Auch hat er einige Abschnitte aus Sсновingers Briefen in seine 1773/74 gedruckte lateinische Briefsammlung aufgenommen.

Verglichen mit GILlERs geistsprühender und witziger Ausdrucksweise wirken Schовingers Briefe eher nüchtern und trocken, wenn man von den

${ }^{39}$ Georg Mathias Gattenhof (1722-1788), Professor in Heidelberg. Schobinger war mit ihm in Göttingen und nennt ihn seinen besten Freund.

${ }^{40}$ HALLer war im Winter 1753 auf der Rathaustreppe gestürzt und hatte sich dabei den Arm gebrochen (nach HiRzel verrenkt). Nach sechs Wochen war er noch nicht wiederhergestellt. 
Bezeugungen seiner Ergebenheit gegenüber HALLER und seinen in Superlativen schwelgenden Lobpreisungen des Meisters absieht. Dabei ist freilich zu bedenken, daß mit dem damaligen, für den Leser nicht immer leicht verständlichen Latein die Entwicklung eines persönlichen originellen Stils kaum möglich war.

Der Wert der Briefe liegt hauptsächlich auf medizinischem Gebiet. Hier erweist sich Sсновіnger als gewissenhafter, auf Fortbildung bedachter, mit der Wissenschaft eng verbundener Arzt, der seinem erhabenen Lehrer Ehre machen will. Vor allem aber scheint er ein guter Beobachter gewesen zu sein, was z.B. daraus hervorgeht, daß ihm die fäulnishemmende Wirkung des Arsens schon hundert Jahre vor der ersten Publikation über den anatomischen Befund bei tödlicher Arsenvergiftung auffiel und daß er die Magenveränderungen bei dieser Vergiftung völlig zutreffend beschrieb (siehe oben). Auch seine Dissertation ist, wie schon erwähnt, eine tüchtige und sorgfältige, über dem Durchschnitt stehende Arbeit, und deshalb ist es zu bedauern, daß er seine späteren Beobachtungen nicht veröffentlicht hat. Aber es entspricht dies seinem bescheidenen und furchtsamen Wesen, das offenbar allen, die ihn näher kannten, auffiel. Seine medizinischen Mitteilungen bezeichnet er selbst als Observatiuncula und Scriptiuncula und einmal entschuldigt er sich wegen seiner Polygraphie. Allem streberhaften Geltungstrieb war er abhold. Er muß aber eine sehr umfassend gebildete Persönlichkeit gewesen sein, denn sonst hätte man ihm nicht den Unterricht in Philosophie an der höheren Lehranstalt anvertraut.

Schoвinger starb am 6.August 1792 und erreichte somit ein Alter von sechsundsechzig Jahren. Er hinterließ wie sein Onkel Giller eine sehr reichhaltige Bibliothek, welche 806 medizinische und naturwissenschaftliche Werke und 1596 Nummern aus anderen Wissensgebieten umfaßte. Nach Schoвingers Tod wurde ein gedruckter Katalog herausgegeben, in welchem die Bücher und Manuskripte in der von dem Besitzer angegebenen Ordnung aufgeführt sind. Auch hat er zum vorneherein die Preise bestimmt. Mit dem höchsten Preis, nämlich 72 Gulden, ist BLAcKwells Herbarium eingeschätzt, Hallers Icones anatomicae mit 28 Gulden und dessen «Physiologie» mit 25 Gulden. Im Vorwort des Katalogs heißt es: «Wer für wenigst $25 \mathrm{fl}$. zusammen nimmt, dem wird noch ein Rabbat (!) von 10\% accordiert. Sollte sich jemand entschließen, diese ganze medicinische Büchersammlung zu übernehmen, so könnte er nicht allein eines beträchtlichen Rabbats versichert seyn, sondern noch den nicht zu verachtenden Vortheil, ein von dem Sel. mit großer Mühe verfertigtes Verzeichnis aller 
Abbildungen derjenigen Pflanzen, die hin und wieder in seiner Bibliothek zerstreut vorkommen, dazu zu bekommen, unter dem Titel: Catal. Figurarum botanicarum quas in suis Libris possidet D. Chr. Schobinger.» Dieser letztere Katalog wirft ein bezeichnendes Licht auf Schobingers Ordnungssinn.

Der Erlös der Bibliothek sollte laut Testament vom 27.April 1784 zu gleichen Teilen Schobingers Witwe und seiner Schwester Maria ElisaBetha Huber-Schobinger zufallen. Ein nicht geringer Teil der Bücher, versehen mit dem Sсновingerschen Wappen, befindet sich jetzt noch in der Stadtbibliothek Vadiana, die von Schobinger mit einem Legat von 50 Gulden bedacht wurde.

Herrn Oberbibliothekar P.-D. Dr. Stranm in Bern danke ich bestens für die Überlassung von Photokopien der Schobingerschen Briefe und Herrn Stadtarchivar Dr.A.Schмid in St. Gallen und Frl. Frieda Schoвinger für ihre Mitteilungen über die Familie Schobinger.

\section{Literatur}

C.Brunner und W. von Muralt, Aus den Briefen hervorragender Schweizer Ärzte des 17. Jahrhunderts, Basel 1919.

Bürgerbuch der Stadt St.Gallen 1950.

Haller, Epistolarum ab eruditis viris ad Alb. Hallerum scriptarum. Latinae, Vol. I-V, Bern $1773 / 74$.

Hirsce, Biographisches Lexikon der hervorragenden Ärzte, Wien/Leipzig 1884.

Hirzel, Albrecht von Hallers Gedichte, Frauenfeld 1882.

P.Jung, Der Stadtarzt Dr. Sebastian Schobinger in St.Gallen, Gesnerus 5, 57 (1948).

D.Langhans, Beschreibung verschiedener Merkwürdigkeiten des Siementhals, eines Theils des Bernergebiets. Nebst einem genauen Bericht über eine neue ansteckende Krankheit, die in diesem Land entstanden. Zürich 1753.

R. Perrola, Das öffentliche Medizinalwesen der Stadt St.Gallen im 17. und 18. Jahrhundert. Zürcher medizin-geschichtliche Abhandlungen, Heft 9, 1926.

Personalia der höheren Lehranstalt in Sankt Gallen. Von ***. St. Gallen 1830. (Der anonyme Verfasser war Pfarrer J. J. BeRnet.)

David Christoph Schobinger, De ortu bilis cysticae et ejus ad vesiculam felleam iinere. Anhang zu der Dissertation von Јон. Heinrich Oschwald. Göttingen 1747.

D. Chr. Schobinger, De telae cellulosae in fabrica corporis humani dignitate, Diss. Göttingen 1748.

Yvonne Thurnheer. Die Stadtärzte und ihr Amt im alten Bern. Berner Beiträge zur Geschichte der Medizin und der Naturwissenschaften, Nr. 4, 1944.

Verzeichnis der hinterlassenen Schobingerschen Bibliothek, St. Gallen 1793.

Wegelin, Briefe des St.Galler Stadtarztes Peter Giller an Albrecht von Haller, Gesnerus 7, 1 (1950). 